Marquette University

e-Publications@Marquette

School of Dentistry Faculty Research and

Publications

Dentistry, School of

3-15-2020

\title{
Incorporation of Functionalized Reduced Graphene Oxide/ magnesium Nanohybrid to Enhance the Osteoinductivity Capability of 3D Printed Calcium Phosphate-based Scaffolds
}

\author{
Meisam Omidi \\ Marquette University \\ Hossein Golzar \\ University of Waterloo \\ Dorsa Mohammadrezaei \\ University of Waterloo \\ Amir Yadegari \\ Marquette University \\ Morteza Rasoulianboroujeni \\ Marquette University
}

See next page for additional authors

Follow this and additional works at: https://epublications.marquette.edu/dentistry_fac

Part of the Dentistry Commons

\section{Recommended Citation}

Omidi, Meisam; Golzar, Hossein; Mohammadrezaei, Dorsa; Yadegari, Amir; Rasoulianboroujeni, Morteza; Hashemi, Mohadeseh; Yazdian, Fatemeh; Shalbaf, Mohammad; and Tayebi, Lobat, "Incorporation of Functionalized Reduced Graphene Oxide/magnesium Nanohybrid to Enhance the Osteoinductivity Capability of 3D Printed Calcium Phosphate-based Scaffolds" (2020). School of Dentistry Faculty Research and Publications. 410.

https://epublications.marquette.edu/dentistry_fac/410 


\section{Authors}

Meisam Omidi, Hossein Golzar, Dorsa Mohammadrezaei, Amir Yadegari, Morteza Rasoulianboroujeni, Mohadeseh Hashemi, Fatemeh Yazdian, Mohammad Shalbaf, and Lobat Tayebi 
Marquette University

e-Publications@Marquette

\section{Dentistry Faculty Research and Publications/College of Dentistry}

This paper is NOT THE PUBLISHED VERSION.

Access the published version via the link in the citation below.

Composites Part B : Engineering, Vol. 185 (March 15, 2020): 107749. DOI. This article is (C Elsevier and permission has been granted for this version to appear in e-Publications@Marquette. Elsevier does not grant permission for this article to be further copied/distributed or hosted elsewhere without the express permission from Elsevier.

\section{Incorporation of Functionalized Reduced Graphene Oxide/magnesium Nanohybrid to Enhance the Osteoinductivity Capability of 3D Printed Calcium Phosphate-based Scaffolds}

\section{Meisam Omidi}

Marquette University School of Dentistry, Milwaukee, WI

Hossein Golzar

Department of Chemistry \& Waterloo Institute for Nanotechnology (WIN), University of Waterloo, Waterloo, Ontario, Canada

Dorsa Mohammadrezaei

Department of Chemistry \& Waterloo Institute for Nanotechnology (WIN), University of Waterloo, Waterloo, Ontario, Canada

Amir Yadegari

Marquette University School of Dentistry, Milwaukee, WI

Morteza Rasoulianboroujeni

Marquette University School of Dentistry, Milwaukee, WI 


\section{Mohadeseh Hashemi}

Department of Biomedical Engineering, The University of Texas at Austin, Austin, TX

\section{Fatemeh Yazdian}

Department of Life Science Engineering, Faculty of New Science and Technologies, University of Tehran, Tehran, Iran

\section{Mohammad Shalbaf}

Protein Research Center, Shahid Beheshti University, GC, Tehran, Iran

\section{Lobat Tayebi}

Marquette University School of Dentistry, Milwaukee, WI

\section{Abstract}

Improving bone regeneration is one of the most pressing problems facing bone tissue engineering (BTE) which can be tackled by incorporating different biomaterials into the fabrication of the scaffolds. The present study aims to apply the 3D-printing and freeze-drying methods to design an ideal scaffold for improving the osteogenic capacity of Dental pulp stem cells (DPSCS). To achieve this purpose, hybrid constructs consisted of 3D-printed Beta-tricalcium phosphate ( $\beta$-TCP)-based scaffolds filled with freeze-dried gelatin/reduced graphene oxide-Magnesium-Arginine (GRMA) matrix were fabricated through a novel green method. The effect of different concentrations of Reduced graphene oxide-Magnesium-Arginine (RMA) $(0,0.25 \%$ and $0.75 \% \mathrm{wt})$ on the morphology, mechanical properties, and biological activity of the 3D scaffolds were completely evaluated. Our findings show that the incorporation of RMA hybrid into the scaffold can remarkably enhance its mechanical features and improve cell proliferation and differentiation simultaneously. Of all scaffolds, $\beta$-TCP/0.25GRMA showed not only the highest ALP activity and cell proliferation after 14 days but it up-regulated bone-related genes and proteins (COL-I, RUNX2, OCN). Hence, the fabricated 3D printed $\beta$-TCP/0.25GRMA porous scaffolds can be considered as a high-potential candidate for BTE.

\section{Keywords}

Magnesium, $\beta$-TCP, Reduced graphene oxide, Arginine, Tissue engineering

\section{Introduction}

The increasing requirement for bone regeneration due to weakening of bone by defects, cancers, trauma, disease, metabolic disorders and war injuries needs enhancing the biological and mechanical properties of scaffold materials [1]. Overall, high osteoconductivity, biodegradability, biocompatibility, mechanical properties and porous interconnected structure are the required features for an ideal bone scaffold [[2], [3], [4]].

Nowadays, bioceramics draw a significant attention of researchers in repairing the bone related defects [5]. Bioceramics such as calcium phosphates have been widely used in bone tissue engineering (BTE) due to their excellent biocompatibility, bioresorbable properties, osteoconductivity, and chemical resemblance to mineral phase of bone $[6,7]$. Among calcium phosphate family, beta tricalcium phosphate $(\beta-T C P)$ grabs more attention in the field of bone replacement due to its desired osteoimmunomodulatory properties which trigger the immune response resulting in accelerated bone regeneration [8,9]. Appropriate osteoimmunomodulatory properties regulate the balance between osteogenesis and osteoclastogenesis that enhance bone healing [10]. Although $\beta$-TCP have a significant bioactivity, its low tensile strength and low fracture toughness limits its application in load bearing [11]. 
Recently, graphene and its derivatives have been introduced as promising materials, which have been found to not only improve mechanical properties of scaffolds, but also induce cell attachment, migration, proliferation and differentiation of stem cells toward osteoblast lineages [[12], [13], [14], [15]]. Graphene, a single twodimensional (2D) layer of conjugated $\mathrm{sp}^{2}$-carbon atoms, have represented desire exceptional physicochemical characteristics for BTE application, including large surface area, high mechanical strength, elasticity modulus, optical and electricity properties [12,[16], [17], [18]]. Graphene oxide (GO) the oxidized form of graphene and Reduced graphene oxide (RGO) produced by reduction the oxygen-containing groups of $\mathrm{GO}$ are two main derivatives of graphene [19]. RGO has the thermal and mechanical properties as same as those of pristine graphene $[20,21]$. Moreover, RGO has shown more ability in electron transferring that may be influential in accelerating osteogenic differentiation [22]. For example, It has been reported that the value of ALP activity as well as the amount of mineralized nodule after 14 and 21 days, respectively, significantly increased by the cells treated with RGO nanoparticles (NPs) compared to those treated with other groups without RGO NPs [23]. However, a few studies reported the toxic effects of RGO toward cells due to its hydrophobic features leading to its aggregating on the cell membrane, which is the main challenge of RGO for biomedical application [12,24]. This challenge can be fixed by functionalizing the surface of RGO by using various polymers such as hyaluronic acid derivatives, dextran, and heparin to make RGO more biocompatible and stable [25]. Among different polymers, l-Arginine (Arg) attracts a significant attention due to its capability in improving the poor stability and hydrophilicity of RGO in aqueous solution which can prevent RGO aggregation [18]. For instance, Hashemi et al. [18] exhibited that Arg functionalized RGO enhanced cell adhesion on the surface of RGO which can be attributed to the positively-charged Arg binding to negatively-charged cell membrane [18].

Although graphene and its derivatives can improve the mechanical strength and promote osteogenesis, they can be chemically modified with other biomaterials to further enhance their osteoconductivity, such as other carbon-based materials [26], hydrogels [27,28], metals [[29], [30], [31]], minerals [16,32,33] and polymers $[34,35]$. Among these materials, metals have attracted the attention of researchers in the field of BTE due to their suitable chemical and physical properties [28]. The chemical functional groups on graphene and its derivatives surfaces such as amino, hydroxyl, and carboxyl groups can act as nucleation sites for them to anchor and prevent their rapid release resulting in improved cell viability, proliferation and osteogenesis [[36], [37], [38]]. Thus, several metals and graphene derivatives nanocomposites have been prepared for biomedical researches, which illustrated synergistic influences over each components $[39,40]$. In a study done by Zhang et al. [36], fabricated GO-Cu nanocomposite was shown to improve cell adhesion, ALP synthesis and OCN expression compared to $\mathrm{GO}$ due to the moderate release of $\mathrm{Cu}$ ions preventing the toxic effect of $\mathrm{Cu}$. Zhang et al. [1] indicated that $\beta$-TCP coated with graphene oxide functionalized with silver nanoparticles (Ag@GO) dramatically accelerated the osteogenesis of rabbit bone marrow stromal cells by enhancing their alkaline phosphatase (ALP) activity and bone-related gene expressions. Among all metals employed in BTE, Mg due to its high mechanical properties, specific strength and elastic modulus close to natural bone, biodegradability and biocompatibility deserve more consideration [41]. However, rapid degradation rate of $\mathrm{Mg}$ is the major challenge preventing them from being applicable in BTE. In this paper, functionalizing RGO with $\mathrm{Mg}$ can not only solve this problem but also induce osteogenic differentiation of stem cells.

The present work aims to use 3D-printing and freeze-drying method to design an ideal scaffold for enhancing osteogenic capacity of Dental pulp stem cells (DPSCs). To achieve this purpose, hybrid constructs consisted of 3D-printed $\beta$-TCP-based scaffolds filled with freeze-dried gelatin/RGO-Mg-Arg (GRMA) matrix was fabricated. The gelatin (Gel) was chosen due to its biodegradability, biocompatibility, cost efficiency and similarity to the organic component of the bone matrix (i.e. collagen) which is commonly utilized in bone replacement applications [[42], [43], [44], [45]]. Additionally, in this study, efforts have focused on the synthesis of RGO-Mg$\operatorname{Arg}(\mathrm{RMA})$ composites via a novel green synthesis method employing $\mathrm{Arg}$ and $\mathrm{NaOH}$ as the reducing and 
stabilizing agent. Ultimately, the physicomechanical and biological properties of the modified $\beta-T C P / G R M A$ hybrid constructs were evaluated for bone regeneration.

\section{Experimental method}

\subsection{Material}

$\beta$-TCP powder, Magnesium Chloride ( $\mathrm{MgCl}_{2}$ ) and I-Arginine (Arg) were obtained from Sigma-Aldrich (USA). Graphit powder (mesh 325), sodium nitrate $\left(\mathrm{NaNO}_{3}\right)$, potassium permanganate $\left(\mathrm{KMnO}_{4}\right)$, potassium persulfate $\left(\mathrm{K}_{2} \mathrm{~S}_{2} \mathrm{O}_{8}\right)$, sodium borohydride $\left(\mathrm{NaBH}_{4}\right)$, hydrochloric acid $(\mathrm{HCL})$ and sulfuric acid $\left(\mathrm{H}_{2} \mathrm{SO}_{4}\right)$ were purchased from Sigma-Aldrich (USA). 1-ethyl-3-[3-dimethylaminopropyl] carbodiimide hydrochloride (EDC), Nhydroxysuccinimide (NHS) and $\mathrm{H}_{2} \mathrm{O}_{2}$ were obtained from Sigma-Aldrich (USA). Gelatin (Type A, from porcine skin, Bioreagent grade) was supplied by Lifecore Biomedical (USA). Sodium Tripolyphospahte (TPP), Alizarin Red S (ARS) dye and Carboxymethylcellulose Sodium salt (CMC) were obtained from Alfa Aesar (USA). Penicillin, streptomycin, Dulbecco's Modified Eagle Medium (DMEM), Phosphate buffered saline (PBS), Amphotericin B, Ascorbic acid and fetal bovine serum (FBS) were supplied by Sigma-Aldrich (UK). Live/dead cellular viability assay kits and Alkialine Phosophatase assay kits were obtained from Thermo-Fisher. DPSCs were obtained from American Type Culture Collection (ATCC).

\subsection{Synthesis of $\mathrm{GO}$}

GO was prepared from graphite powder using modified Hummer's method [19]. The typical procedure was as follows, graphite powder $(3 \mathrm{~g}), \mathrm{K}_{2} \mathrm{~S}_{2} \mathrm{O}_{8}(2.5 \mathrm{~g})$, and $\mathrm{P}_{2} \mathrm{O}_{5}(2.5 \mathrm{~g})$ were mixed together and then $12 \mathrm{~mL}$ concentrated $\mathrm{H}_{2} \mathrm{SO}_{4}$ was added under vigorous stirring at $80{ }^{\circ} \mathrm{C}$ for $4.5 \mathrm{~h}$. Prior to gradually adding $\mathrm{KMnO}_{4}(15 \mathrm{~g})$, $\mathrm{H}_{2} \mathrm{SO}_{4}(120 \mathrm{~mL})$ was added to prepared graphite oxide. To prevent overheating and explosion, the temperature was kept below $20{ }^{\circ} \mathrm{C}$ during the addition of $\mathrm{KMnO}_{4}$. Subsequently, the reaction mixture was stirred at $40{ }^{\circ} \mathrm{C}$ for $2 \mathrm{~h}$ followed by adding $750 \mathrm{~mL}$ deionized water and $20 \mathrm{~mL} \mathrm{H} \mathrm{O}_{2}$. Finally, the resulting mixture was washed with $1 \mathrm{~L} \mathrm{HCl}(1 \% \mathrm{v} / \mathrm{v})$ and deionized water $(1 \mathrm{~L})$, respectively. $\mathrm{GO}$ was obtained after the reaction solution had been dried, dispersed and purified using dialysis bag (7000 Da) [19].

\subsection{Synthesize of RMA hybrid}

RMA hybrid was synthesized through hydrothermal reaction [46]. The typical procedure was as follows, $12 \mathrm{mg}$ $\mathrm{NaOH}$ was dispersed in $20 \mathrm{ml} \mathrm{GO}$ solution $(0.5 \mathrm{mg} / \mathrm{ml})$ under constant stirring. Subsequently, $36 \mathrm{mg} \mathrm{MgCl}_{2}$ was added to the reaction mixture to provide $\mathrm{Mg}^{2+}$, followed by adding $10 \mathrm{mg} \mathrm{Arg}$. The reaction mixture was stirred for $15 \mathrm{~min}$ and then incubated at $130^{\circ} \mathrm{C}$ for $24 \mathrm{~h}$.

\subsection{Characterization of GO and RMA hybrid}

The surface morphology of the GO and RMA hybrid was examined using transmission electron microscopy (TEM, Zeiss, EM10C, $80 \mathrm{KV}$ ) and atomic force microscopy (AFM, SPM VESCO). FTIR spectra were recorded by a Spectrum RX I, PerkinElmer FT-IR. X-ray diffractions (XRD, PANalytical, X'Pert PRO MPD, Netherlands) were performed by using a $\mathrm{Cu} K \alpha$ radiation source with a step size of $0.02(2 \theta) / \mathrm{Sec}$. The RMA hybrid was analyzed by Energy Dispersive X-ray spectroscopy on an SEM (Thermoscientific SEM-EDX). The zeta potential analysis of the samples was measured using a Malvern ZEN 3600 zetasizer.

\subsection{Preparation of ink}

The ceramic based ink is composed of beta-tricalcium phosphate powder ( $\beta$-TCP), carboxymethylcellulose (CMC), sodium tripolyphosphate (TPP), and deionized water. The paste was prepared according to the previously reported procedure [43]. At first, $0.5 \mathrm{~g}$ of TPP and $0.075 \mathrm{~g}$ of CMC were thoroughly mixed in $5 \mathrm{ml}$ deionized water. After that, $15 \mathrm{~g}$ of $\beta$-TCP was added and mixed completely for $10 \mathrm{~min}$. Finally, $500 \mu \mathrm{l}$ of 
deionized water added to the obtained mixture and defoamed at $2000 \mathrm{rpm}$ using centrifugal mixture (THINKY, Japan) for 1 min and loaded into a plotting cartridge (Nordson, USA).

\subsection{Fabrication of scaffolds}

The ceramic-based scaffolds were fabricated with the desired geometry, structure, and size using the 3D bio plotter (Envision TEC, Germany). The desired pattern of scaffolds was designed using a CAD/CAM software. The printing parameters including pressure, temperature, and speed of printing were optimized to be 1.5-1.7 bar, $26^{\circ} \mathrm{C}$, and $3-5 \mathrm{~mm} / \mathrm{s}$, respectively. The scaffolds were 3D-printed through a layer by layer deposition using a plotting needle via an inner diameter of $400 \mu \mathrm{m}$ to fabricate the discs with diameter of $15 \mathrm{~mm}$, thickness of $2 \mathrm{~mm}, 90^{\circ}$ lay down patterns, and 0.6-0.8 $\mathrm{mm}$ distance between strands. The scaffolds were 3D-printed on an alumina plate which already polished with a thin layer of paraffin to easily remove each scaffold after 3Dprinnting. Subsequently, the fabricated scaffolds were air died over night. Next, the scaffolds were sintered using a high temperature muffle furnace through a sequential heating program. The temperature of furnace was raised from room temperature to $600{ }^{\circ} \mathrm{C}$ with a rate of $4{ }^{\circ} \mathrm{C} / \mathrm{min}$ and incubated at $600{ }^{\circ} \mathrm{C}$ for $1 \mathrm{~h}$. Then, the temperature increased to $1100^{\circ} \mathrm{C}$ with a rate of $5{ }^{\circ} \mathrm{C} / \mathrm{min}$ and kept for $4 \mathrm{~h}$.

\subsection{Fabrication of $\beta$-TCP/GRMA hybrid constructs}

The hybrid constructs were prepared according to the previous reported procedure [47]. At first, the prepared RMA hybrid described above mixed with gelatin to obtain a homogenous solution and then the resulting solution was filled into the pores of the 3D-printed $\beta$-TCP scaffolds, followed by immersing in liquid nitrogen for $10 \mathrm{~min}$. Subsequently, the $\beta$-TCP/GRMA hybrid constructs was obtained by lyophilizing the frozen mixture.

Finally, the stability of the $\beta$-TCP/GRMA hybrid construct increased by crosslinking them with EDC/NHS solution.

\subsection{Characterization of scaffolds}

\subsubsection{Morphological characterization}

3D laser scanning digital microscope (Olympus LEXT OLS) were used to assess the scaffold's morphology and surface roughness. Different sites of scaffolds $(n=6)$ were randomly selected to measure various microstructural features including roughness and dimension of the pores size, thickness of strands and distance between them by utilizing image software(LEXT OLS 4000).

\subsubsection{XRD analysis}

The pure TCP and $\beta$-TCP/GRMA hybrid constructs were characterized using XRD analysis. The powder was then sieved to $150 \mu \mathrm{m}$ particles (Endecotts, UK).

\subsubsection{Mechanical properties}

The compressive modulus and strength of the $\beta$-TCP/GRMA hybrid constructs were measured utilizing a mechanical testing machine (Shimadzu, Japan) with a $5 \mathrm{kN}$ load cell, and a cross-head speed of $1.0 \mathrm{~mm} \mathrm{~min}^{-1}$. The compression of the cylindrical samples $(n=5)$ continued until they fractured. Finally, the mean compressive strength and modulus were calculated by using the data obtained.

\subsection{Cell viability and adhesion}

Cell viability was assessed by using Presoblue assay (PB, Invitrogen, USA), according to the manufacturer's instructions, after 1,7 , and 14 days of tissue culture. The spectrophotometric plate reader (Infinite ${ }^{\circledR}$ M200,TECAN, USA) at $560 \mathrm{~nm}$ excitation and $590 \mathrm{~nm}$ emission was used to measure the fluorescence. After 14 days, the samples were imaged using SEM to examine cell attachment and proliferation. All scaffolds were washed with PBS, prior to fixing with $3 \%$ of glutaraldehyde, and dehydrated with gradual ethanol washes in various concentrations of ethanol (50\%-100\%). Then, samples were sputter-coated with gold ( $20 \mathrm{~nm})$ and imaged using SEM (Philips XL-20, USA) with an acceleration voltage of $15 \mathrm{kV}$. 


\subsection{Alkaline phosphatase activity}

ALP activity of cells cultured on scaffolds was evaluated using an ALP assay kit (Abcam, USA) according to the manufacturer's instructions. Briefly, at day 7 and $14,50 \mu \mathrm{L}$ lysate was reacted with $150 \mu \mathrm{L} p$-nitrophenyl phosphate (p-NPP) at $37^{\circ} \mathrm{c}$ for $30 \mathrm{~min}$. After that, the reaction was terminated by adding stop solution and samples were measured for absorbance at $405 \mathrm{~nm}$ using a micro plate reader (Synergy HTX, BioTEK).

\subsection{Real-time polymerase chain reaction (RT-PCR)}

Cell seeded onto scaffolds for osteogenesis-related genes expression analysis including osteocalcin (OCN), Type I collagen (COL-I) and Runt-related transcription factor 2 (RUNX-2) at day 7 and 14. RNA was extracted utilizing isolate II RNA Mini Kit (BioLine) according to the manufacturer's instructions and its purity and concentration was evaluated using the NanoDrop 1000 Spectrophotometer (ThermoScientific) at 260/280 nm. Complementary DNA (cDNA) was generated utilizing High Capacity RNA-to-cDNA Kit (Applied Biosystems, UK), according to the manufacturer's instructions. qPCR analysis was performed as described [6]. Briefly, in each PCR reaction $5 \mu l$ equal volumes of TaqMan universal PCR Master Mix, $0.5 \mu \mathrm{l}$ B2M internal controls (Applied Biosystems), $3.5 \mu \mathrm{l}$ nuclease free water, and equal volumes of CDNA and PCR primers were mixed in a total volume of $10 \mu \mathrm{l}$ and recorded through Rotor- Gen Q Software (QIAGEN). Each PCR reaction was performed in triplicate.

\subsection{Statistical analysis}

All data were expressed as mean and standard deviation (mean \pm SD), One-way analysis of variance (ANOVA) and two-tailed t-test were utilized to assess the statistical significance with $P$-value $<0.05$.

\section{Result and discussion}

\subsection{Characterization of GO and RMA}

RMA nanohybrid was successfully synthesized and characterized. Arg was covalently linked to RGO to not only enhance cell adhesion via electrostatic interactions but also stabilize the RGO sheets in cell culture and prevent its aggregation [46] and then MgNPs were grown on the surface of RGO. AFM was employed to evaluate the morphologies of synthesized GO (Fig. 1a). AFM images exhibited that the lateral width and average thickness of synthesized GO was about $80-850 \mathrm{~nm}$ and $4.86 \mathrm{~nm}$, respectively. Covalent grafting of Arg was confirmed by FTIR through the presence of Arg absorption peaks at approximately 3265 and $1322 \mathrm{~cm}^{-1}$ which may be assigned to $\mathrm{N}-\mathrm{H}$ and $\mathrm{C}-\mathrm{N}$ bonds, respectively (Fig. 1b) [19]. Also, the decrease in the intensity of some characteristic peaks of $\mathrm{GO}$ such as $\mathrm{C}=\mathrm{C}\left(1610 \mathrm{~cm}^{-1}\right)$ can be attributed to the interaction between MgNPs and RGO surface, as well as the partial reduction of GO [48]. Moreover, after successful conjugation of Arg to GO, the zeta potential of GO inverted to $+52.75 \pm 4.45$ which also confirm the result were obtained by FTIR. 

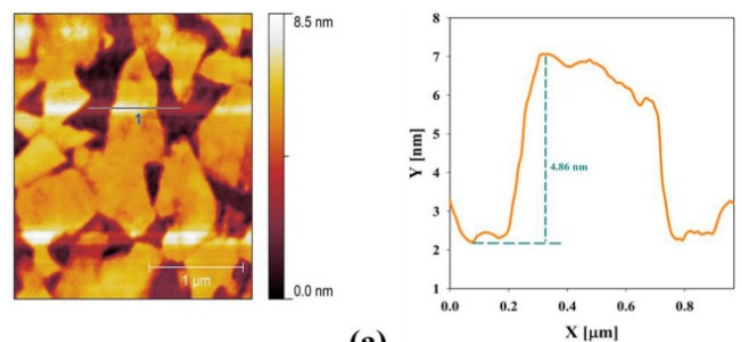

(a)

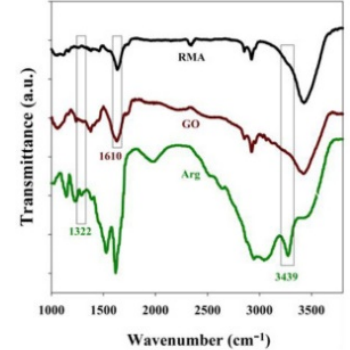

(b)

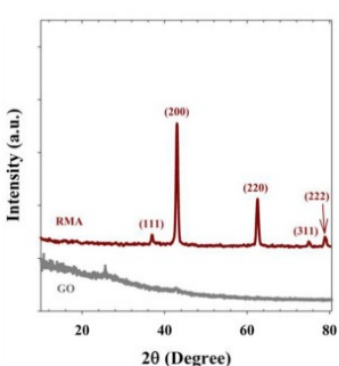

(c)

Fig. 1. (a)The AFM image of GO, (b) FTIR spectra of Arg, GO, RMA, (c) XRD patterns of GO.

Due to the alteration of the crystal behavior of GO after functionalizing with MgNPs, XRD analysis can be used as an efficient approach to confirm formation of MgNPs in the RGO structure, after reduction [49]. As shown in Fig. $1 \mathrm{c}$, the XRD pattern of $\mathrm{GO}$ showed a distinctive peak at $2 \theta \approx 9.8^{\circ}$ disappeared in the XRD pattern of RMA as a result of $\mathrm{GO}$ reduction [21]. Besides, the diffraction peaks of the nanohybrid at $38.11^{\circ}, 43.35^{\circ}, 62.43^{\circ}, 75.2^{\circ}$ and $78.91^{\circ}$ assigned to (111), (200), (220), (311) and (222) planes, respectively, indicate a pattern of the cubic MgNPs in the hybrid structure [49]. Similar results were reported by Du et al. [50].

SEM-EDX was also employed to characterize the microstructure as well as the elemental composition of the hybrid (Fig. 2a). As can be seen in Fig. 2a, the surface of RMA was covered with MgNPs which is confirmed through elemental analysis. Analyzing the elemental composition of the selected area demonstrated that it contains $\mathrm{Mg}$, oxygen and carbon elements with 41.1, 48.9, $10.0 \mathrm{wt} \%$. For further morphological information, TEM images of GO and RMA were also examined (Fig. $2 b$ and c). Fig. $2 b$ exhibited the folding structure of GO without any nanoparticles or impurity while Fig. 2c showed the formation of MgNPs on the surface of RGO sheets with appropriate distribution. These observations are in a good agreement with SEM-EDX results which implies that the RMA nanohybrid was successfully synthesized. 


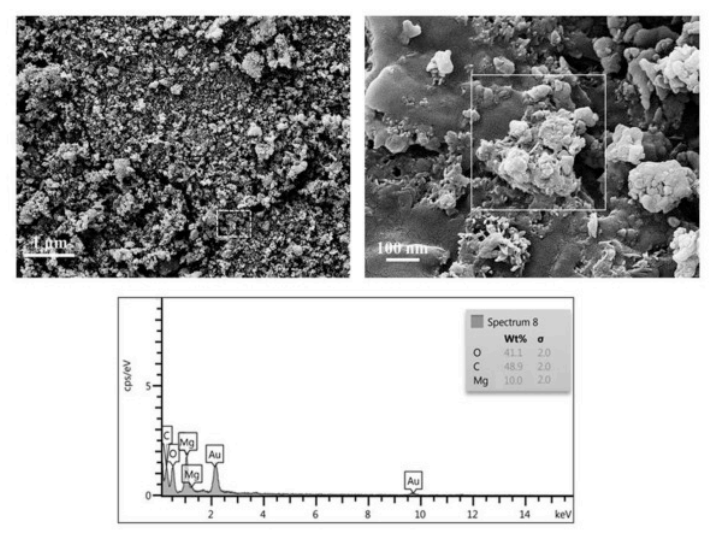

(a)

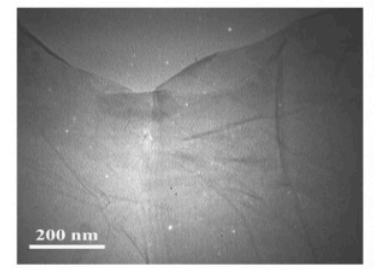

(b)

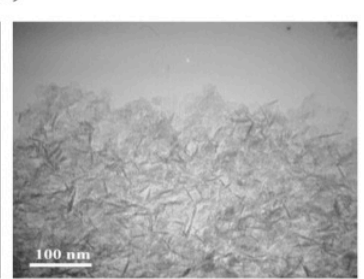

(c)

Fig. 2. (a) The SEM-EDX of RMA, (b) TEM of GO and (c) RMA

\subsection{Characterization of scaffold}

To investigate the crystalline structure of pure TCP and sintered $\beta-T C P, X R D$ analysis was performed (See Supplementary 1, Fig. S1). As can be seen in Fig. S1, pure and sintered $\beta$-TCP indicated approximately the same distinctive peaks. Similar results were reported by Almela et al. [6].

The porosity and pores interconnectivity of the bone can have a remarkable effect on the cellular behavior by providing a suitable microenvironment for bone growth and vascularization [[51], [52], [53]]. Hence, several studies investigated the optimum pore size and reported that the optimum value is about $400 \mu \mathrm{m}$ which can vary due to the cell source, size and nutrition [54]. Besides, due to the fact that the total porosity of human cancellous bone is in the range of $30 \%-90 \%$ so that the scaffold which has porosity within this range can be considered as an efficient scaffold for bone tissue regeneration [55]. To evaluate the morphology and surface roughness of the fabricated scaffolds, the 3D-printed $\beta$-TCP/GRMA hybrid constructs was examined by 3D laser microscopy (Fig. 3). As shown in Fig. 3, the pore size of the $\beta$-TCP/GRMA scaffolds was about $425 \pm 25 \mu \mathrm{m}$ which is in a good agreement with optimized values mentioned above. Moreover, the strands thickness and distance between stands was measured about 0.5 and $1 \mathrm{~mm}$, respectively, which were in the range of the trabecular struts in the cancellous bone [55]. 

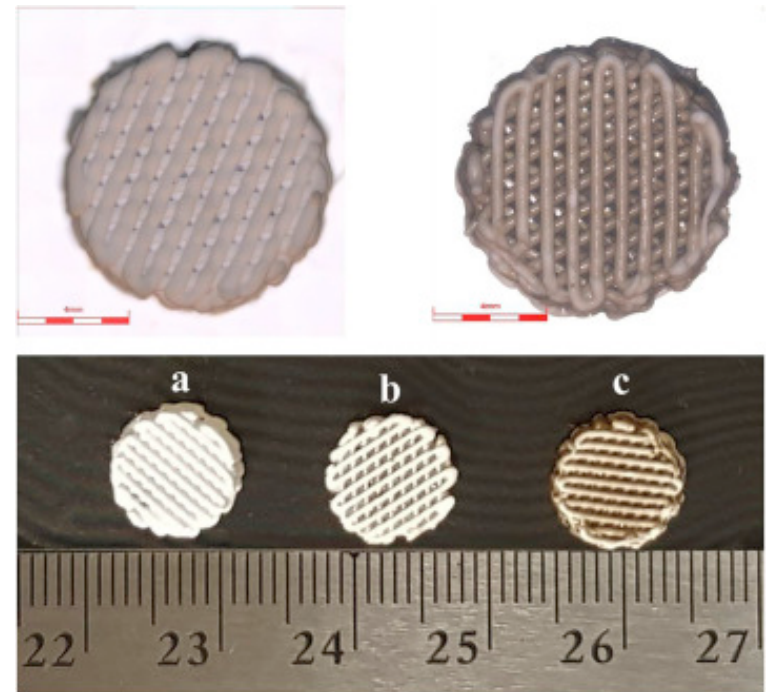

Fig. 3. The 3D laser scanning images of the 3D-printed TCP scaffold.

The biological activity of the biomaterials can significantly enhance by increasing their surface roughness. The unique nanotopography of the graphene based materials may improve the surface roughness of bone scaffolds which can effectively enhance the biological activity of the scaffold by providing a suitable area for cellular and protein interactions [15]. Hence, the surface roughness of $\beta$-TCP, $\beta-$ TCP/0.25GRMA, and $\beta-$ TCP/0.75GRMA was measured and they were $0.62 \pm 0.13,1.6 \pm 0.19$, and $3.7 \pm 0.2$, respectively, which clearly showed that surface roughness increased by increasing the concentration of GRMA hybrid. In a study done by Wu et al. [56], the correlation between surface roughness and bone cells response was evaluated and they reported the optimum average roughness was in the range of $0.8-1.0 \mu \mathrm{m}$.

Mechanical strength should be considered as an influential factor in 3D scaffold designing. Young's modulus is known as an important index of mechanical characteristics were measured for $\beta$-TCP/GRMA hybrid constructs through stress-strain curve (Fig. 4). As can be seen in Fig. 4, by increasing the concentration of GRMA, the young's modulus increases too which can be attributed to the inherent mechanical properties of MgNPs and RGO. Indeed, the presence of amine group of Arg on the surface of RGO resulted in preventing the restacking of RGO sheets which can cause good dispersion of RGO sheets. This can significantly improve the mechanical strength of the $\beta$-TCP scaffolds and thus provide cells with a condition in which they can grow inside the scaffold to gradual replacement of regenerated bone tissue [41]. 


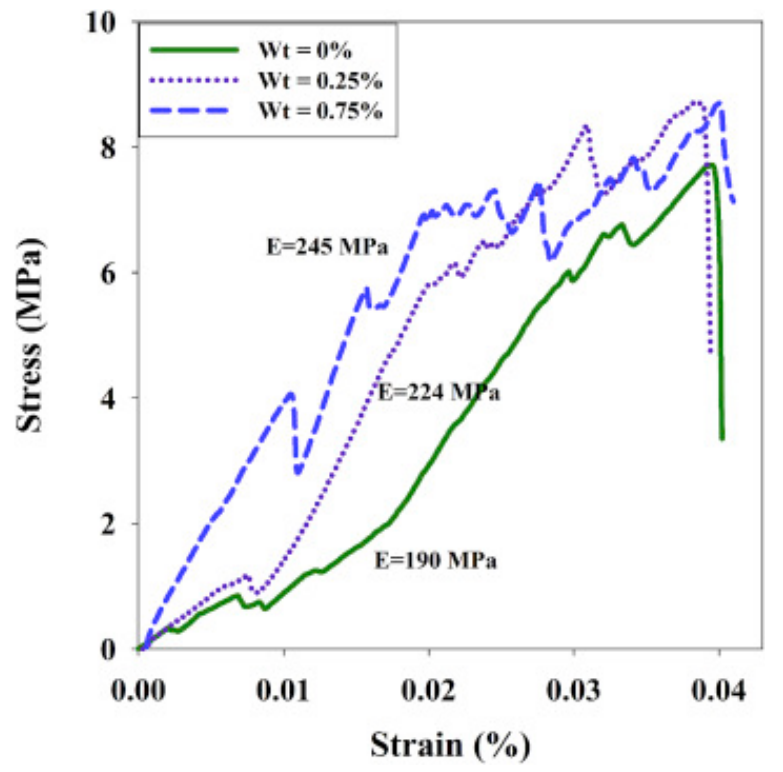

Fig. 4. The stress-strain curve of $\beta$-TCP, $\beta$-TCP/0.25GRMA, and $\beta$-TCP/0.75GRMA $(n=5)$.

\subsection{Proliferation and Attachment}

Cell attachment and proliferation within the scaffold are usually known as a primary steps of cell seeding, and therefore, have a remarkable influence on modulating the cell responses such as cell proliferation and differentiation [57]. In several studies reported that the presence of graphene and its derivatives can contribute significantly to the acceleration of cell adhesion and spreading [[58], [59], [60]]. The results of the present study showed that despite significantly improved proliferation of DPSCs cultured on the $\beta$-TCP/0.75GRMA than those cultured on $\beta$-TCP and $\beta$-TCP/0.25GRMA on 7th day, after 14 days, $\beta$-TCP/0.25GRMA exhibited higher cell proliferation compared with other groups (Fig. 5a). For further investigation, the cells seeded on the $\beta$ TCP/GRMA scaffolds were stained for live/dead assay (Fig. 5b, 5c, and $5 \mathrm{~d}$ ). As can be seen in Fig. $5 c$, the density of live cells significantly increased with time which showed that the cells attached and grew better on the surface of $\beta$-TCP/0.25GRMA scaffold compared to other scaffolds.

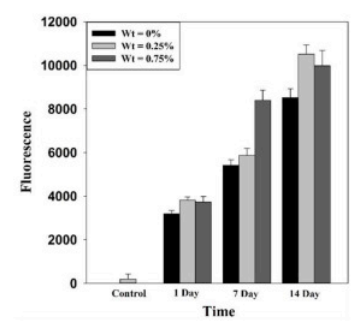

(a)

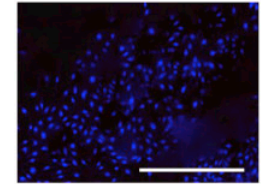

(b)

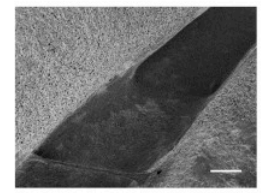

(e)

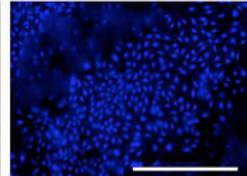

(c)

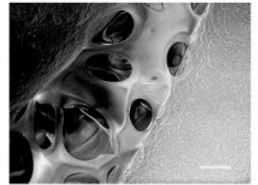

(f)

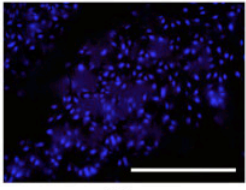

(d)

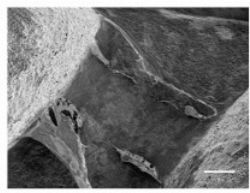

(g) 
Fig. 5. Proliferation of DPSCs on the 3D-printed scaffolds at day 1, 7 and 14 by PB assay (a), Fluorescence micrographs of DPSCs on the $\beta$-TCP (b), $\beta$-TCP/0.25GRMA (c), and $\beta$-TCP/0.75GRMA (d), at day 14 , the SEM images for $\beta$-TCP (e), $\beta$-TCP/0.25GRMA (f), and $\beta$-TCP/0.75GRMA (g) after 14 days. $n=3$ per scaffold, $* \mathrm{P}<0.05$; $* * P<0.01$; relative to control.

Moreover, to investigate the primary attachment of cells, the SEM images for 3D-printed $\beta$-TCP/GRMA scaffold constructs seeded with DPSCs after 14 days were captured (Fig. $5 \mathrm{e}, 5 \mathrm{f}$ and $5 \mathrm{~g}$ ). As can be seen, the incorporation of the freeze-dried 0.25GRMA matrix into the $\beta$-TCP scaffold resulted in dramatically high attachment and proliferation of DPSCs, which represents the enhanced seeding efficiency onto the hybrid constructs.

Promoted proliferation and adhesion of cells cultured on $\beta$-TCP/GRMA can be attributed to the high biocompatibility of suitable dose of RMA and Gel. Gel due to its hydrophilic feature as well as the existence of glycine, proline, and hydroxyproline could enhance cell adhesion and proliferation [33]. While in RMA, Arg by generating amine functional groups on RGO surface could improve its hydrophilicity and biocompatibility. In study done by Kumar et al. [61] it was observed that addition of amine-functionalized graphene oxide (AGO) in Poly( $\varepsilon$-Caprolactone) (PCL) resulted in more adsorbed cell-adhesive proteins; thus, AGO-PCL represented higher cell adhesion compared to GO-PCL and RGO-PCL. Hydrophilic substrate can provide a surface letting cells to attach, proliferate and grow better on them [62]. In other words, more hydrophilicity leads to more protein adsorption playing a significant role in improving cell attachment through better formation of focal adhesions (FAs) [12]. FAs are a type of adhesive contact between cells and ECM which can modulate cells attachment and migration $[63,64]$. Moreover, the interaction between the positively-charged Arg and negatively-charged cell membrane could enhance DPSCs adhesion [46]. The roughness of RMA is also of importance in this improvement which can affect the cellular adhesion and proliferation through providing more surface areas for more interaction with proteins and cells.

$\mathrm{Mg}$ ions play an essential role in regulating the cell functions, particularly by the activation of integrins. $\mathrm{Mg}^{2+}$ ions initiate conformational activation of integrins to binding ligand to sites on the integrin $\alpha$-chain. Therefore, addition of $\mathrm{Mg}$ into tissue engineering constructs could effectively promote cell-scaffold interactions and cell functions $[65,66]$. However, the major challenge for using $\mathrm{Mg}$ is its high corrosion rate resulting in the rapid release of hydrogen gas and formation of gas pockets around the scaffold which can interrupt the tissue regeneration $[67,68]$. Moreover, this degradation increased the alkalinity of the culture, which may lead to the death of cells. Consequently, controlling the degradation rate of $\mathrm{Mg}$ to achieve the perfect balance of ions is essential for applying magnesium for BTE [69].

Previously, it has been demonstrated that functionalization of graphene and its derivatives can present nucleation sites for metals to anchor and prevent their rapid release [38,70]. For instance, Kumar et al. [71] illustrated that RGO decorated with strontium (Sr) showed enhanced mineral deposition and cell proliferation compared to RGO without $\mathrm{Sr}$ owing to the sustain release of $\mathrm{Sr}^{2+}$. Therefore, in this study it is possible that RGO with its negatively-charged functional groups improved the stability of the positively-charged $\mathrm{Mg}$ ions on its surface [72] and thus prevent their rapid release which can ultimately improve the cellular behavior as well as the mechanical properties of scaffold.

\subsection{Osteogenic differentiation}

In order to evaluate osteoinductive ability of 3D printed $\beta$-TCP, $\beta$-TCP/0.25GRMA and $\beta$-TCP/0.75GRMA, ALP activity of DPSCs as one of the early osteogenic differentiation markers was assessed over two weeks. As can be seen in Fig. 6a, after 7 and 14 days, the ALP activity of the DPSCs cultured on $\beta$-TCP/0.25GRMA scaffolds was higher than that on $\beta$-TCP/0.75GRMA and pure $\beta$-TCP scaffolds, indicating the positive influence of GRMA at a suitable dose. To further confirm the DPSCs differentiation, the mRNA expressions of cultured cells were 
evaluated by RT-PCR (Fig. 6). After 7 and 14 days of culturing, DPSCs on $\beta$-TCP/0.25GRMA expressed an obviously higher level of the osteogenic gene (COL-I, Runx2, OCN) than those on the $\beta$-TCP/0.75GRMA and pure $\beta$-TCP scaffolds (Fig. 6b, $c$ and d). Though after 14 days culture, the gene levels of COL-I decreased, gene expression levels of this gene was still significantly higher in $\beta$-TCP/0.25GRMA group than those in $\beta$-TCP group. All these results supported the successful and facilitated differentiation of DPSCs into osteoblasts on $\beta T C P / 0.25 G R M A$ due to the synergetic effect of Gel and appropriate dose of RMA.
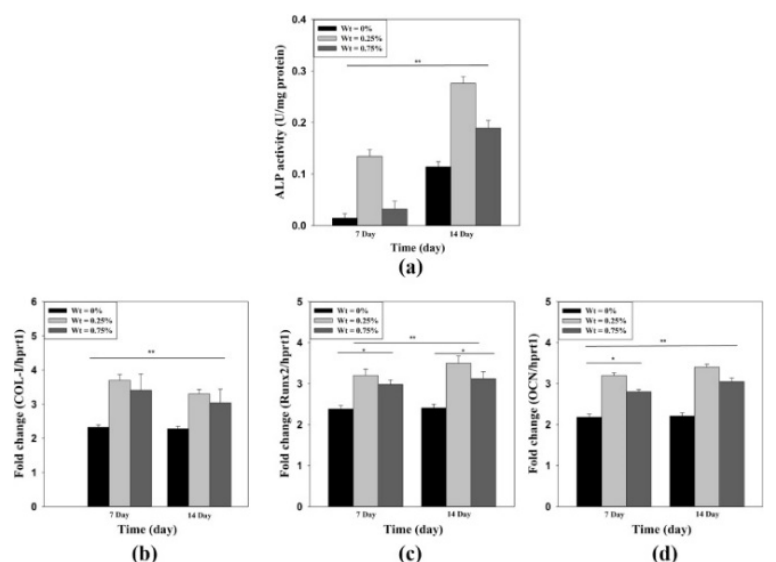

Fig. 6. ALP activity of DPSCs after cultivation on the $\beta$-TCP scaffolds (a) Relative mRNAs expression of COL-I (b), Runx2 (c) and OCN (d) on DPSCs on the $\beta$-TCP scaffolds at 7 and 14 days. $n=3$ per scaffold, $* P<0.05 ;{ }^{*} \mathrm{P}<0.01$; relative to control.

It has been demonstrated that proteinaceous composition of Gel presents multiple amino-acid side chains (aspartates and glutamates) for better interaction with DPSCs and ions for mineralization [73]. In a study performed by Liu et al. [73] The surface of GO was modified by Gel resulted in a bioinspired surface with negatively-charged carboxylate groups, which were observed to increase the adsorption of calcium ions, and hence acted as nucleation sites for hydroxyl apatite (HA) mineralization. Moreover, RGO functionalized with Arg and Mg showed positive effects on cell osteogenesis. Indeed, amine functional groups on the RGO surface provided by Arg could serve as binding point for calcium and phosphate ions [74], thus enhanced the mineralization of HA [75]. Mg ions were also observed that could accelerate mineralization. $\mathrm{Mg}$ depletion adversely influences all stages of skeletal metabolism, resulting in a decline in osteoblast activities and bone brittleness. Hence, the incorporation of Mg ions $\beta$-TCP-based scaffold is of great interest for the development of bone scaffolds [76].

Finally, synergistic action of RMA as well as ECM mimicking chemical composition provided by Gel would have simultaneously improve the differentiation of stem cells toward osteoblastic lineage even without the presence of osteogenic medium.

\section{Conclusion}

In summary, RMA hybrid nanostructures were synthesized through hydrothermal method and fully characterized via various analyses such as FTIR, XRD, SEM-EDX, and TEM. After that, different $\beta$-TCP-based scaffolds containing various contents of RMA hybrid $(0,0.25$ and $0.75 \% \mathrm{wt})$ and gelatin were fabricated using 3D printing method. The results show that the addition of RMA not only improved the mechanical properties but it also enhance the cell proliferation which can be attributed to the amine groups of Arg. Additionally, of all scaffolds $\beta$ TCP/0.25GRMA showed the highest ALP activity and cell proliferation after 14 days which can be rooted in the synergistic effect of sustain release of $\mathrm{Mg}$ ions and amine groups existing on the surface of the scaffold. Overall, we recommend that the novel $\beta$ TCP/0.25GRMA can be employed as an effective scaffold in 
BTE due to its high potential in inducing cell behaviors such as viability, proliferation, differentiation which are proven in this study.

\section{Acknowledgements}

This research received no specific grant from any funding agency in the public, commercial, or not-for-profit sectors.

\section{References}

1. Y. Zhang, D. Zhai, M. Xu, Q. Yao, H. Zhu, J. Chang, et al. 3D-printed bioceramic scaffolds with antibacterial and osteogenic activity. Biofabrication, 9 (2017), p. 25037,

2. C. Chua. Review the design of scaffolds for use in tissue engineering. Part II. Rapid Prototyping Techniques, 8 (2002)

3. H.R.R. Ramay, M. Zhang. Biphasic calcium phosphate nanocomposite porous scaffolds for load-bearing bone tissue engineering 25 (2004), pp. 5171-5180

4. L. Gonc, G.E. Crippa, PT De Oliveira. Pore size regulates cell and tissue interactions with PLGA - CaP scaffolds used for bone engineering (2012), pp. 155-162,

5. M. Logesh, A. Marimuthu, A.M. Ballamurugan. Fabrication of graphene incorporated biphasic calcium phosphate composite and evaluation of impact of graphene in the in-vitro biomineralization process Mater Chem Phys, 232 (2019), pp. 75-81

6. T. Almela, I.M. Brook, K. Khoshroo, M. Rasoulianboroujen. Bioprinting Simulation of cortico-cancellous bone structure by 3D printing of bilayer calcium phosphate-based sca ff olds Bioprinting, 6 (2017), pp. 1-7,

7. Q.Z. Chen, A.R. Boccaccini. Improved mechanical reliability of bone tissue engineering (Zirconia) scaffolds by Electrospraying. 1539 (2006), pp. 1534-1539

8. C. Shuai, Y. Li, G. Wang, W. Yang, S. Peng, P. Feng. Surface modification of nanodiamond: toward the dispersion of reinforced phase in poly-L-lactic acid scaffolds. Int J Biol Macromol, 126 (2019), pp. 11161124

9. C. Shuai, S. Li, S. Peng, P. Feng, Y. Lai, C. Gao. Biodegradable metallic bone implants Mater Chem Front, 3 (2019), pp. 544-562

10. M. Yazdimamaghani, M. Razavi, D. Vashaee, L. Tayebi Development and degradation behavior of magnesium scaffolds coated with polycaprolactone for bone tissue engineering Mater Lett, 132 (2014), pp. 106-110,

11. P. Feng, P. Wu, C. Gao, Y. Yang, W. Guo, W. Yang, et al. A multimaterial scaffold with tunable properties: toward bone tissue repair Adv Sci, 5 (2018), p. 1700817

12. D. Mohammadrezaei, H. Golzar, M.R. Rad, M. Omidi, H. Rashedi, F. Yazdian, et al. Review Article in vitro effect of graphene structures as an osteoinductive factor in bone tissue engineering A Syst Rev (2018), pp. 2284-2343

13. W. Nie, C. Peng, X. Zhou, L. Chen, W. Wang, Y. Zhang, et al. Three-dimensional porous scaffold by selfassembly of reduced graphene oxide and nano-hydroxyapatite composites for bone tissue engineering Carbon N Y, 116 (2017), pp. 325-337

14. H. Xie, M. Chua, I. Islam, R. Bentini, T. Cao, J.C. Viana-Gomes, et al. CVD-grown monolayer graphene induces osteogenic but not odontoblastic differentiation of dental pulp stem cells Dent Mater, 33 (2017), pp. e13-21

15. Z. Tian, L. Huang, X. Pei, J. Chen, T. Wang, T. Yang, et al. Electrochemical synthesis of three-dimensional porous reduced graphene oxide film: preparation and in vitro osteogenic activity evaluation Colloids Surfaces B Biointerfaces, 155 (2017), pp. 150-158

16. C. Fu, H. Bai, J. Zhu, Z. Niu, Y. Wang, J. Li, et al. Enhanced cell proliferation and osteogenic differentiation in electrospun PLGA/hydroxyapatite nanofibre scaffolds incorporated with graphene oxide PLoS One, 12 (2017), Article e0188352 
17. E.-S. Kang, D.-S. Kim, I.R. Suhito, S.-S. Choo, S.-J. Kim, I. Song, et al. Guiding osteogenesis of mesenchymal stem cells using carbon-based nanomaterials Nano Converg, 4 (2017), p. 2

18. M. Hashemi, M. Omidi, B. Muralidharan, H. Smyth, M.A. Mohagheghi, J. Mohammadi, et al.Evaluation of the photothermal properties of a reduced graphene oxide/arginine nanostructure for near-infrared absorption. ACS Appl Mater Interfaces, 9 (2017), pp. 32607-32620

19. S. Shahmoradi, H. Golzar, M. Hashemi Optimizing the nanostructure of graphene oxide/silver/arginine for effective wound healing Nanotechnology, 29 (2018), p. 475101

20. O.C. Compton, S.T. Nguyen Graphene oxide, highly reduced graphene oxide, and graphene: versatile building blocks for carbon-based materials Small, 6 (2010), pp. 711-723

21. H. Golzar, F. Yazdian, M. Hashemi, M. Omidi, D. Mohammadrezaei, H. Rashedi, et al. Optimizing the hybrid nanostructure of functionalized reduced graphene oxide/silver for highly efficient cancer nanotherapy New J Chem, 42 (2018), pp. 13157-13168

22. O. Akhavan, E. Ghaderi, E. Abouei, S. Hatamie, E. Ghasemi Accelerated differentiation of neural stem cells into neurons on ginseng-reduced graphene oxide sheets Carbon N Y, 66 (2013), pp. 395-406

23. L. Jin, J.H. Lee, O.S. Jin, Y.C. Shin, M.J. Kim, S.W. Hong, et al. Stimulated osteogenic differentiation of human mesenchymal stem cells by reduced graphene oxide J Nanosci Nanotechnol, 15 (2015), pp. 7966-7970

24. M. Kalbacova, A. Broz, J. Kong, M. Kalbac Graphene substrates promote adherence of human osteoblasts and mesenchymal stromal cells Carbon N Y, 48 (2010), pp. 4323-4329

25. P. Feng, J. He, S. Peng, C. Gao, Z. Zhao, S. Xiong, et al. Characterizations and interfacial reinforcement mechanisms of multicomponent biopolymer based scaffold Mater Sci Eng C, 100 (2019), pp. 809-825

26. X. Yan, W. Yang, Z. Shao, S. Yang, X. Liu Graphene/single-walled carbon nanotube hybrids promoting osteogenic differentiation of mesenchymal stem cells by activating p38 signaling pathway Int J Nanomed, 11 (2016), pp. 5473-5484

27. Sunny C. Patel, B.S. Owais Alam Osteogenic differentiation of human adipose derived stem cells on chemically crosslinked carbon nanomaterial coatings J Biomed Mater Res A (2017)

28. L.R. Jaidev, S. Kumar, K. Chatterjee. Multi-biofunctional polymer graphene composite for bone tissue regeneration that elutes copper ions to impart angiogenic, osteogenic and bactericidal properties Colloids Surfaces B Biointerfaces, 159 (2017), pp. 293-302

29. J. Qiu, H. Geng, D. Wang, S. Qian, H. Zhu, Y. Qiao, et al. Layer-number dependent antibacterial and osteogenic behaviors of graphene oxide electrophoretic deposited on titanium ACS Appl Mater Interfaces, 9 (2017), pp. 12253-12263

30. L. Han, H. Sun, P. Tang, P. Li, C. Xie, M. Wang, et al. Mussel-inspired graphene oxide nanosheetenwrapped $\mathrm{Ti}$ scaffolds with drug-encapsulated gelatin microspheres for bone regeneration Biomater Sci, 3 (2018), pp. 39-48

31. N. Dubey, K. Ellepola, F.E.D. Decroix, J.L.P. Morin, A. Castro Neto, C.J. Seneviratne, et al. Graphene onto medical grade titanium: an atom-thick multimodal coating that promotes osteoblast maturation and inhibits biofilm formation from distinct species Nanotoxicology (2018), pp. 1-16

32. Q. Wang, Y. Chu, J. He, W. Shao, Y. Zhou, K. Qi, et al. A graded graphene oxide-hydroxyapatite/silk fibroin biomimetic scaffold for bone tissue engineering Mater Sci Eng C, 80 (2017), pp. 232-242

33. S. Saravanan, C. Anjali, M. Vairamani, T.P. Sastry, K.S. Subramanian, N. Selvamurugan. Scaffolds containing chitosan, gelatin and graphene oxide for bone tissue regeneration in vitro and in vivo. Int J Biol Macromol (2016

34. Q. Yao, J. Jing, Q. Zeng, T.L. Lu, Y. Liu, X. Zheng, et al. Bilayered BMP2 eluting coatings on graphene foam by electrophoretic deposition: electroresponsive BMP2 release and enhancement of osteogenic differentiation. ACS Appl Mater Interfaces, 9 (2017), pp. 39962-39970

35. P. Feng, S. Peng, P. Wu, C. Gao, W. Huang, Y. Deng, et al. A nano-sandwich construct built with graphene nanosheets and carbon nanotubes enhances mechanical properties of hydroxyapatitepolyetheretherketone scaffolds. Int J Nanomed, 11 (2016), pp. 3487-3500 
36. W. Zhang, Q. Chang, L. Xu, G. Li, G. Yang, X. Ding, et al. Graphene oxide-copper nanocomposite-coated porous CaP scaffold for vascularized bone regeneration via activation of Hif-1 $\alpha$. Adv Healthc Mater, 5 (2016), pp. 1299-1309

37. J. Qiu, J. Guo, H. Geng, W. Qian, X. Liu. Three-dimensional porous graphene nanosheets synthesized on the titanium surface for osteogenic differentiation of rat bone mesenchymal stem cells. Carbon $\mathrm{N}$ Y, 125 (2017), pp. 227-235

38. C. Xu, X. Wang, L. Yang, Y. Wu. Fabrication of a graphene-cuprous oxide composite. J Solid State Chem, 182 (2009), pp. 2486-2490

39. A. Cao, Z. Liu, S. Chu, M. Wu, Z. Ye, Z. Cai, et al. A facile one-step method to produce craphene-CdS quantum dot nanocomposites as promising optoelectronic materials. Adv Mater, 22 (2010), pp. 103106

40. Y. Zou, Y. Wang. NiO nanosheets grown on graphene nanosheets as superior anode materials for Li-ion batteries. Nanoscale, 3 (2011), p. 2615

41. C. Shuai, B. Wang, Y. Yang, S. Peng, C. Gao. 3D honeycomb nanostructure-encapsulated magnesium alloys with superior corrosion resistance and mechanical properties. Compos B Eng, 162 (2019), pp. $611-620$

42. H. Liao, K.T. Shalumon, K. Chang, C. Sheu, J. Chen. And conductive factors in gelatin-based cryogels. J Mater Chem B (2016)

43. M.N. Khan, J.M.M. Islam, M.A. Khan. Fabrication and characterization of gelatin-based biocompatible porous composite scaffold for bone tissue engineering. 2 (2012), pp. 1-9

44. X. Liu, L.A. Smith, J. Hu, P.X. Ma. Biomaterials Biomimetic nanofibrous gelatin/apatite composite scaffolds for bone tissue engineering. Biomaterials, 30 (2009), pp. 2252-2258

45. C. He, F. Zhang, L. Cao, W. Feng, K. Qiu, Y. Zhang, et al. Rapid mineralization of porous gelatin scaffolds by electrodeposition for bone tissue engineering. J Mater Chem, 22 (2012), pp. 2111-2119,

46. M. Hashemi, A. Yadegari, G. Yazdanpanah, S. Jabbehdari, M. Omidi, L. Tayebi .Functionalized R9reduced graphene oxide as an efficient nano-carrier for hydrophobic drug delivery. RSC Adv, 6 (2016), pp. 74072-74084

47. F. Fahimipour, E. Dashtimoghadam, M. Rasoulianboroujeni, M. Yazdimamaghani, K. Khoshroo, M. Tahriri , et al. Collagenous matrix supported by a 3D-printed scaffold for osteogenic differentiation of dental pulp cells. Dent Mater, 1-12 (2017)

48. S. Gurunathan, J.W. Han, J.H. Park, E. Kim, Y.-J. Choi, D.-N. Kwon, et al. Reduced graphene oxide - silver nanoparticle nanocomposite : a potential anticancer nanotherapy. Int J Nanomed, 10 (2015), pp. 62576276

49. J.R. Lee, J.Y. Bae, W. Jang, J.-H. Lee, W.S. Choi, H.Y. Koo. Magnesium hydroxide nanoplate/graphene oxide composites as efficient adsorbents for organic dyes. RSC Adv, 5 (2015), pp. 83668-83673

50. Du F, Yang W, Zhang F, Tang C, Liu S, Yin L, et al. Enhancing the heat transfer Efficiency in graphene epoxy nanocomposites using a magnesium oxide - graphene hybrid structure n.d. doi:10.1021/acsami.5b03196.

51. A.S. Brydone, D. Meek, S. Maclaine. Proc Inst Mech Eng H J Eng Med (2010)

52. Q.L. Loh, C. Choong. Three-dimensional scaffolds for tissue engineering applications: role of porosity and pore size. Tissue Eng B Rev (2012

53. M. Bohner, Y. Loosli, G. Baroud, D. Lacroix. Deciphering the link between architecture and biological response of a bone graft substitute. Acta Biomater, 7 (2011), pp. 478-484

54. J. Rouwkema, N.C. Rivron, CA Van Blitterswijk. Vascularization in tissue engineering. (2008

55. V. Karageorgiou, D. Kaplan. Porosity of 3D biomaterial scaffolds and osteogenesis. Biomaterials, 26 (2005), pp. 5474-5491

56. $\mathrm{C}$. Wu, M. Chen, T. Zheng, X. Yang. Effect of surface roughness on the initial response of MC3T3-E1 cells cultured on polished titanium alloy. 26 (2015), pp. 155-164

57. F.J.O. Brien. Biomaterials \& scaffolds Every day thousands of surgical procedures are performed to replace. Mater Today, 14 (2011), pp. 88-95 
58. Y. Liu, T. Chen, F. Du, M. Gu, P. Zhang, X. Zhang, et al. Single-layer graphene enhances the osteogenic differentiation of human mesenchymal stem cells in vitro and in vivo. J Biomed Nanotechnol, 12 (2016), pp. 1270-1284

59. M. Nair, D. Nancy, A.G. Krishnan, G.S. Anjusree, S. Vadukumpully, S.V. Nair. Graphene oxide nanoflakes incorporated gelatin-hydroxyapatite scaffolds enhance osteogenic differentiation of human mesenchymal stem cells. Nanotechnology, 26 (2015), p. 161001

60. S. Zhang, Q. Yang, W. Zhao, B. Qiao, H. Cui, J. Fan, et al. In vitro and in vivo biocompatibility and osteogenesis of graphene-reinforced nanohydroxyapatite polyamide66 ternary biocomposite as orthopedic implant material. Int J Nanomed, 11 (2016), pp. 3179-3189

61. S. Kumar, S. Raj, E. Kolanthai, A.K. Sood, S. Sampath, K. Chatterjee. Chemical functionalization of graphene to augment stem cell osteogenesis and inhibit biofilm formation on polymer composites for orthopedic applications. ACS Appl Mater Interfaces, 7 (2015), pp. 3237-3252

62. H.S. Jung, T. Lee, I.K. Kwon, H.S. Kim, S.K. Hahn, C.S. Lee. Surface modification of multipass caliberrolled ti alloy with dexamethasone-loaded graphene for dental applications. ACS Appl Mater Interfaces, 7 (2015), pp. 9598-9607

63. L. Wang, G. Zhou, H. Liu, X. Niu, J. Han, L. Zheng, et al. Nano-hydroxyapatite particles induce apoptosis on MC3T3-E1 cells and tissue cells in SD rats. Nanoscale, 4 (2012), p. 2894

64. C.J. Wilson, R.E. Clegg, D.I. Leavesley, M.J. Pearcy. Mediation of biomaterial-cell interactions by adsorbed proteins: a review. Tissue Eng, 11 (2005), pp. 1-18

65. A.P. Yamniuk, H.J. Vogel. Calcium- and magnesium-dependent interactions between calcium- and integrin-binding protein and the integrin alphallb cytoplasmic domain. Protein Sci, 14 (2005), pp. 14291437

66. H. Zreiqat, C.R. Howlett, A. Zannettino, P. Evans, G. Schulze-Tanzil, C. Knabe, et al. Mechanisms of magnesium-stimulated adhesion of osteoblastic cells to commonly used orthopaedic implants. J Biomed Mater Res, 62 (2002), pp. 175-184

67. G. Song. Control of biodegradation of biocompatable magnesium alloys. Corros Sci, 49 (2007), pp. 1696-1701

68. H. Man, K.W.K. Yeung, K. On, V. Tam, P.K. Chu, K.D.K. Luk, et al. Biomaterials A biodegradable polymerbased coating to control the performance of magnesium alloy orthopaedic implants. Biomaterials, 31 (2010), pp. 2084-2096

69. T.Y. Nguyen, S. Garcia, C.G. Liew, H. Liu. Effects of magnesium on growth and proliferation of human embryonic stem cells. (2012), pp. 723-726

70. Y.W. Wang, A. Cao, Y. Jiang, X. Zhang, J.H. Liu, Y. Liu, et al. Superior antibacterial activity of zinc oxide/graphene oxide composites originating from high zinc concentration localized around bacteria. ACS Appl Mater Interfaces, 6 (2014), pp. 2791-2798

71. S. Kumar, K. Chatterjee. Strontium eluting graphene hybrid nanoparticles augment osteogenesis in a 3D tissue scaffold. Nanoscale, 7 (2014), pp. 2023-2033

72. N. Zhang, H. Qiu, Y. Si, W. Wang, J. Gao. Fabrication of highly porous biodegradable monoliths strengthened by graphene oxide and their adsorption of metal ions. Carbon N Y, 49 (2011), pp. 827837

73. H. Liu, J. Cheng, F. Chen, D. Bai, C. Shao, J. Wang, et al. Biomimetic and cell-mediated mineralization of hydroxyapatite by carrageenan functionalized graphene oxide. ACS Appl Mater Interfaces, 6 (2014), pp. 3132-3140

74. D. Depan, B. Girase, J.S. Shah, R.D.K. Misra. Structure-process-property relationship of the polar graphene oxide-mediated cellular response and stimulated growth of osteoblasts on hybrid chitosan network structure nanocomposite scaffolds. Acta Biomater, 7 (2011), pp. 3432-3445

75. W.C. Lee, C.H.Y.X. Lim, H. Shi, L.A.L. Tang, Y. Wang, C.T. Lim, et al. Origin of enhanced stem cell growth and differentiation on graphene and graphene oxide. ACS Nano, 5 (2011), pp. 7334-7341, 
76. A.R. Toibah, I. Sopyan, M. Hamdi, S. Ramesh. Development of magnesium-doped biphasic calcium phosphatethrough Sol-Gel method. 4th Kuala Lumpur Int. Conf. Biomed. Eng. 2008. Springer Berlin Heidelberg, Berlin, Heidelberg (2008), pp. 314-317 